\title{
Use of a Neural Net to Model the Impact of Optical Coherence Tomography Abnormalities on Vision in Age- Related Macular Degeneration
}

DOI:

10.1016/j.ajo.2017.10.015

\section{Document Version}

Accepted author manuscript

Link to publication record in Manchester Research Explorer

Citation for published version (APA):

Aslam, T. M., Mahmood, S., Ali, Z. C., Ahmad, N. A., Thorell, M. R., \& Balaskas, K. (2017). Use of a Neural Net to Model the Impact of Optical Coherence Tomography Abnormalities on Vision in Age-Related Macular

Degeneration. American Journal of Ophthalmology, 185, 94-100. https://doi.org/10.1016/j.ajo.2017.10.015

Published in:

American Journal of Ophthalmology

\section{Citing this paper}

Please note that where the full-text provided on Manchester Research Explorer is the Author Accepted Manuscript or Proof version this may differ from the final Published version. If citing, it is advised that you check and use the publisher's definitive version.

\section{General rights}

Copyright and moral rights for the publications made accessible in the Research Explorer are retained by the authors and/or other copyright owners and it is a condition of accessing publications that users recognise and abide by the legal requirements associated with these rights.

\section{Takedown policy}

If you believe that this document breaches copyright please refer to the University of Manchester's Takedown Procedures [http://man.ac.uk/04Y6Bo] or contact uml.scholarlycommunications@manchester.ac.uk providing relevant details, so we can investigate your claim.

\section{OPEN ACCESS}




\section{Accepted Manuscript}

Use of a Neural Net to Model the Impact of Optical Coherence Tomography Abnormalities on Vision in Age-Related Macular Degeneration

Tariq M. Aslam, Haider R. Zaki, Sajjad Mahmood, Zaria C. Ali, Nur A. Ahmad, Mariana R. Thorell, Konstantinos Balaskas

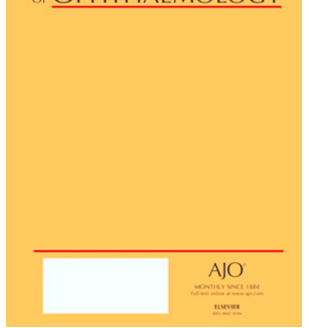

PII:

S0002-9394(17)30446-4

DOI:

10.1016/j.ajo.2017.10.015

Reference: $\quad$ AJOPHT 10295

To appear in: American Journal of Ophthalmology

Received Date: 10 August 2017

Revised Date: 17 October 2017

Accepted Date: 22 October 2017

Please cite this article as: Aslam TM, Zaki HR, Mahmood S, Ali ZC, Ahmad NA, Thorell MR, Balaskas $\mathrm{K}$, Use of a Neural Net to Model the Impact of Optical Coherence Tomography Abnormalities on Vision in Age-Related Macular Degeneration, American Journal of Ophthalmology (2017), doi: 10.1016/ j.ajo.2017.10.015.

This is a PDF file of an unedited manuscript that has been accepted for publication. As a service to our customers we are providing this early version of the manuscript. The manuscript will undergo copyediting, typesetting, and review of the resulting proof before it is published in its final form. Please note that during the production process errors may be discovered which could affect the content, and all legal disclaimers that apply to the journal pertain. 


\section{Abstract}

Purpose: To develop a neural network for the estimation of visual acuity from optical coherence tomography (OCT) images of patients with neovascular age related macular degeneration and to demonstrate its use to model the impact of specific controlled OCT changes on vision.

Design: Artificial Intelligence (neural network) study.

Methods: We assessed 1400 OCT scans of patients with neovascular age related macular degeneration (AMD). 15 physical features for each eligible OCT as well as patient age were used as input data and corresponding recorded visual acuity as the target data to train, validate and test a supervised neural network. We then applied this network to model the impact on acuity of defined OCT changes in subretinal fluid, subretinal hyperreflective material and loss of external limiting membrane integrity.

Results: 1,210 eligible OCT scans were analysed resulting in 1210 data points which were each 16 dimensional. A ten layer feed-forward neural network with one hidden layer of 10 neurons was trained to predict acuity and demonstrated a root mean square error of 8.2 letters for predicted compared to actual visual acuity and a mean regression coefficient of 0.85 . A virtual model using this network demonstrated the relationship of visual acuity to specific, programmed changes in OCT characteristics. When external limiting membrane (ELM) is intact, there is a shallow decline in acuity with increasing sub-retinal fluid but a much steeper decline with equivalent increasing sub-retinal hyperreflective material. When ELM is not intact, all visual acuities are reduced. Increasing subretinal hyperreflective material or subretinal fluid in this circumstance reduces vision further still, but with a smaller gradient than when ELM is intact.

Conclusions: The supervised machine learning neural network developed is able to generate an estimated visual acuity value from OCT images in a population of patients with AMD. These findings should be of clinical and research interest in macular degeneration, for example in estimating visual prognosis or highlighting the importance of developing treatments targeting more visually destructive pathologies. 
Use of a Neural Net to Model the Impact of Optical Coherence Tomography Abnormalities on Vision in Age-Related Macular Degeneration.

Tariq M Aslam ${ }^{1,2}$, Haider R Zaki ${ }^{1}$, Sajjad Mahmood ${ }^{2}$, Zaria C Ali ${ }^{2}$, Nur A Ahmad $^{3}$, Mariana R Thorell ${ }^{2,4}$, Konstantinos Balaskas ${ }^{1,2}$.

${ }^{1}$ School of Pharmacy and Optometry, Faculty of Biology, Medicine and Health,The University of Manchester, Manchester, United Kingdom.

${ }^{2}$ Manchester Royal Eye Hospital, NHS Central Manchester University Hospitals, Manchester, United Kingdom.

${ }^{3}$ Department of Optometry, Faculty of Health Sciences, Universiti Teknologi Mara Puncak Alam, 42300, Selangor, Malaysia.

${ }^{4}$ Department of Ophthalmology Hospital Banco de Olhos de Porto Alegre, Porto Alegre, Brazil

Short Title; Neural Network to model OCT abnormality impact on vision

Corresponding Author:

Tariq M Aslam, Manchester Royal Eye Hospital, Oxford Road, Manchester, UK

Phone number: +441612765325

Fax number: +441612765325

E-mail: Tariq.aslam@cmft.nhs.uk 


\section{Introduction}

Modern-day management of retinal disease is increasingly supported by the use of advanced and multi-modal imaging technologies. Optical coherence tomography (OCT) is one particularly crucial technology that has allowed for more accurate characterisation of retinal conditions such as neovascular age-related macular degeneration (AMD). ${ }^{1-3}$ In AMD structural OCT measures such as macular thickness have been instrumental in confirming response to anti-vegf agents ${ }^{1}$ and further research has explored the clinical importance and relevance of other OCT features, such as pigment epithelial detachments, ${ }^{2}$ subretinal fluid ${ }^{3,4}$ and subretinal fibrosis. ${ }^{5}$ Studies that specifically assess the relationship between such structural imaging findings and visual function ${ }^{6-8}$ provide particularly important information for both research and clinical practice.

For example, detailed knowledge of the visual impact of subretinal fibrosis ${ }^{9}$ may impact on the motivation, design and interpretation of research studies investigating new emerging treatments for AMD. ${ }^{10}$ Similarly, a demonstration that subretinal fibrosis is associated with particularly poor visual acuity, ${ }^{11}$ but that small amounts of persistent subretinal fluid may be tolerated without harm, ${ }^{4}$ is pertinent in clinical practice to both counselling and treatment planning for patients with AMD.

The application of modern computing techniques such as artificial intelligence might help provide further insight into the impact of OCT structural imaging abnormalities on vision. One such form of artificial intelligence is based upon the training of systems of interlinked simple processing 'elements' or artificial 'neurons', known as neural networks. As every neuron can be linked with many other neurons ${ }^{12}$ these networks provide a powerful ability to represent both linear and non-linear relationships directly from the data being modelled. ${ }^{13}$ Supervised neural networks are those that are trained to produce outputs in response to sample inputs and these are particularly well suited to modelling dynamic systems and predicting events. ${ }^{14}$

The purpose of this study was firstly to initialise, train and validate a supervised neural network to determine a value for visual acuity from a patient's OCT structural data and age. Secondly we applied this network to model the impact of user-defined and controlled OCT changes on vision, namely thickness of subretinal fluid and subretinal hyperreflective material as well as external limiting membrane integrity.

\section{Methods}

OCT images with corresponding age and visual acuity data were collected from patients who had attended Manchester Royal Eye Hospital macular treatment centre between the dates of Jan 2013- Jan 2017. The project was institutionally approved as retrospective anonymised data analysis by the 
Central Manchester Foundation Trust Research and Development office and adhered to the Declaration of Helsinki.

Patients who had been diagnosed with neovascular AMD and had no ocular co-morbidity that could impact on visual acuity were included in the study. Exclusion criteria were OCTs technically compromised due to significant tilting or decentration or cases in which the image quality was too poor make necessary detailed measurements.

For each patient a horizontal B scan OCT image had been acquired with a spectral domain OCT machine (Topcon 3D OCT-2000, Tokyo Japan). The macular protocol used consisted of $512 \times 128$ raster scan covering an area of $6 \times 6 \mathrm{~mm}$ centred on the fovea. The assessing ophthalmologist determined the horizontal B scan image closest to the centre of the fovea. Then, masked to other patient information, the ophthalmologist used semi-automated techniques to measure strictly defined physical characteristics of this OCT image, at the centre point of the fovea, using the calliper function in the proprietary OCT viewer software as required.

The OCT characteristics chosen to be assessed were incorporated on the basis of achieving a practical balance of three factors. Firstly, In order to allow for broadest application, input data was limited to that which could be acquired simply from standard spectral-domain OCT scans. Secondly our protocol required for the chosen measures to be ones that are feasible for an expert observer to determine objectively and accurately in thousands of images. Finally we attempted to measure key features that clinical experience and research have suggested make most significant impact on vision. In total 15 measurement inputs were chosen as the most pragmatic OCT features to incorporate.

Firstly, thickness measurements in micrometres of the following anatomical structures were taken at the centre of the fovea for each OCT image; neuroretina, subretinal hyper-reflective material, subretinal fluid, sub-pigment epithelial hyperreflective material and sub-pigment epithelial fluid. Choroidal thickness was also measured as well as the extent of choroidal shadow as a representation of the level of atrophy. For this measurement we determined in micrometres the depth of increased choroidal transmittance of illuminating beam in the foveal area relative to surrounding unaffected areas. The assessor then determined the number of intra retinal cysts in the area of foveal depression and graded foveal anatomy as being concave, flat or convex inner surface. Presence or absence of an intact external limiting membrane/ellipsoid zone complex (ELM) was recorded. Finally, the retinal thickness in the central foveal zone and in each of the four surrounding quadrants was measured automatically by the proprietary OCT software.

All this OCT data was compiled along with the age of the patient. In total 16 separate input measures were recorded for each patient, all feasible for replication in a standard clinical setting. All image assessment was done by a single ophthalmologist with retinal experience $(\mathrm{HZ})$ overseen by an expert group of retinal specialists (TA, KB, SM). 
The recorded information on OCT characteristics and patient age was used to provide the input dataset to a neural network. In order to equalise impact on training in a neural net, all these feature vectors were standardised to have a mean of 0 and standard deviation of 1.The recorded visual acuity of the corresponding patient at the time the OCT was taken was used to provide the target value defining the desired network output. Visual acuity in each case had been measured as numbers of letters read on an ETDRS chart as part of routine clinical care using a standardised departmental protocol with habitual correction and added pinhole. Our aim was to assess over 1,000 eligible images for the study, a sample size in excess of similar published studies which developed trained supervised neural nets. ${ }^{15-17}$

The available data was divided into training, validation and test sets based upon time of accession. The training set (70\%) was used to build the model, with the technique of scaled conjugate gradient backpropagation. In backpropagation, after comparing the computed activation with its target, the associated error for that pattern is calculated and is distributed back to all units in the previous layer. ${ }^{18}$ All network development was performed by the first author (TA). A separate validation set (15\% of the data) was used to compare the performances of the prediction algorithms that were created based on the training set. It was used to estimate how well the model had been trained and to estimate model properties. The algorithm that had the best performance was then chosen.

A final discrete test set (15\% of the data) was used to evaluate the final model algorithm. In order to maximise use of available data, OCTs of both eyes of patients as well as from multiple visits were permitted in the development stages. For the validation and test set only a single OCT scan was permitted per patient to ensure a statistically valid and unbiased assessment of the final derived neural net. The root mean square of error and regression coefficients between target values (actual visual acuity) and neural network output (predicted visual acuity) were calculated for the training, validation and testing datasets respectively (MATLAB R2015aSP1).

Finally we used our developed neural network to simulate the impact on vision of specific and controlled OCT changes. This was done using a separate function written in MATLAB (R2015 aSP1) calling upon the newly developed neural network algorithm. This function allowed for the determination of the effect on vision of precise designated OCT features. We chose from clinical interest to simulate the impact of subretinal fluid and of subretinal hyperreflective material, both with and without intact external limiting membrane.

The separate software function set each of the component OCT input measures to be the mean values of our study sample. The software function input these values into the developed neural net algorithm to determine an expected associated visual acuity for this virtual OCT scan. Keeping most of these values stable, the function then varied the level of the values representing subretinal fluid. At each change in level of subretinal fluid, the 
function called upon the developed neural net to determine an associated expected visual acuity. The consequent sequence of outputs represents the impact on visual acuity of changes in amount subretinal fluid. In turn, for each level of subretinal fluid, the function also calculated expected vision for a range of levels of subretinal hyperreflective material. The whole process was repeated with the value of external limiting membrane set to be intact and then with external limiting membrane set to be not intact. The data from all modelled visual acuity outcomes were incorporated into three dimensional plots of levels of subretinal fluid and hyperreflective material against expected associated vision.

\section{Results}

In total, we assessed 1400 sequential scans of patients who were undergoing treatment with intravitreal Ranibizumab or Aflibercept for wet age-related macular degeneration. 190 were excluded due to hazy media or technically unsatisfactory detail with 1210 scans consequently eligible for analysis. All 16 input datasets for each of these 1210 eligible scans was completed, resulting in a total of 19,360 variables that were manually input.

$847(70 \%)$ of the eligible OCTs were used for network training. These were derived from 123 eyes of 113 patients. 182 separate scans (15\%) were used for parameter optimisation/ validation. For the final evaluation study, a further separate cohort of 182 patients (15\% of total) was used. For these last two studies, only one scan of one eye was incorporated from each patient.

We developed a neural net using this input and visual acuity as the desired output. This consisted of a feed-forward network with 10 sigmoid hidden layer neurons and linear output neurons. Regression, $\mathrm{R}$ values represent the association between neural net outputs and actual target visual acuity. The resultant regression coefficient from our model on the training set was 0.866 , for the validation set 0.872 and for the final testing set the regression coefficient was 0.852. This data is displayed graphically in Figure 1.

In order to expose the most important input features we used a process of sequential feature selection. Our algorithm added features sequentially and performed 10 -fold cross validation by repeatedly calling our function with different OCT candidate features. At each stage the feature was chosen which would minimise the distance or loss of predicted values from actual values. Ultimately we generated a ranking of predictive value of features. A summary of input data characteristics along with forward feature selection predictive value ranking is provided in Table 1.

The resultant mean squared error (average squared difference between outputs and targets) from our final model on the training set was 67.0 , for the validation set 69.4 and for the final testing set the mean squared error was 67.5. Thus the final root mean square deviation of the neural net on the testing data set was 8.21 letters. 
For modelling of impact of structural changes we have produced threedimensional line graphs presented in Figures 2 and 3. The graphs show that for all levels of subretinal fluid and subretinal hyperreflectivity, visual acuity is superior in patients with intact external limiting membrane on OCT. In patients with intact external limiting membrane (Figure 2), visual acuity falls sharply with increases in subretinal hyperreflectivity but falls only slightly with similar increases in subretinal fluid. This relatively small decrease in acuity from subretinal fluid is similar with all co-existing levels of subretinal hyperreflectivity. In patients with no intact external limiting membrane (Figure 3 ), visual acuity still falls sharply with increases in subretinal hyperreflectivity, but there appears to be even less impact on acuity from subretinal fluid than when external limiting membrane is intact.

\section{Discussion}

In developing this neural net we aspired to input OCT data that would be most clinically relevant, determined using semi-automated measurements defined by strict criteria. However, this also exposed the study to significant sources of potential error as the measures were not fully automated and objective measurement and localisation of physical aspects of OCT such as hyperreflective material was on occasions challenging. Ensuring measurements were centred on the fovea centre also involved potential for error and further weaknesses came from measurement of atrophy by assessment of choroidal shadow after assessing en-face colour image - This could be more accurately done through other imaging modalities such as auto-fluorescent imaging. Finally, improvements to the model might also be made by using additional data such as previous OCT findings or other imaging modalities.

The model demonstrates a root mean squared error of 8.21 letters. This equates to a $95 \%$ confidence of prediction to within approximately 16.4 letters of recorded visual acuity. Although seemingly broad, the accuracy of Va prediction should be considered in comparison to the low inter-session reliability of visual acuity measurement in AMD. ${ }^{19}$ Our own research team's assessment of coefficient of repeatability for distance LogMAR was 14.9 letters when measured under rigorous controlled trial standards of optometric refracted acuity. ${ }^{20}$ For this study we used standard non-refracted acuities measured as per normal clinical practice and so the reliability of visual acuity measurement is likely to be broader still. Schmidt-Erfurth et al. ${ }^{21}$ conducted an elegant study which used fully automated image analysis To evaluate the potential of machine learning to predict best-corrected visual acuity (BCVA) outcomes in patients receiving standardized ranibizumab therapy for AMD from spectral-domain (SD) OCT. Although their system was superior in terms of its automation, this restricted certain key OCT structural components from being incorporated such as external limiting membrane patency or measures of subretinal fibrosis. At baseline, their random forest regression model resulted in an accuracy of $R^{2}=0.21$ with a root mean square error of 11.4 letters. Thus considering the limits inherent to reliability of vision testing, our 
neural network model achieves an acceptable level of accuracy which may be favourably compared to other techniques. This accuracy value is relevant to predicting an individual's vision. However, the model we developed is used to represent more systematic behaviours of vision with respect to controlled changes in input OCT features.

The impact of changes in ELM is starkly demonstrated as the graph of visual acuity measures with ELM not intact (Figure 3) demonstrates significantly lower levels of acuity for all amounts of subretinal fluid or fibrosis than when ELM is intact (Figure 2). Typically visual acuity dropped by approximately 1015 letters. This reflects clinical and experimental evidence on the importance of the integrity of this structure to visual prognosis.

The three-dimensional plots (Figures 2 \& 3) demonstrate that, as expected from clinical experience and experimental studies, subretinal hyperreflective material has a more significant impact on visual acuity than subretinal fluid. This finding occurred irrespective of amount of subretinal fluid or patency of ELM. The impact appears to be linear. This finding underlines the importance of subretinal hyperreflective material or fibrosis as an independent factor causing visual morbidity and the need for more management options to tackle this aspect of AMD.

The impact of subretinal fluid is less marked. It has a broadly linear but relatively small impact on acuity when ELM is intact. This finding concurs with previous authors who have suggested that small amounts of SRF can be tolerated. ${ }^{4}$ Subretinal fluid has even less impact when the ELM is not intact with the integrity of ELM a much more dominant factor in affecting acuity.

Weaknesses inherent in our neural net include those due to the limited subcategorisations of OCT structure that we measured. More detailed stratification such as of hyperreflective material components might improve the algorithm in future. Other OCT findings such as hyperreflective dots or outer retinal tubulation as well as findings from multimodal imaging use could also be incorporated. Our algorithms are applicable only to patients with AMD as neural nets may not generalize well if tested away from the training set distribution. The range of values in our training set however, was very large, with many of the patients having normal values for many of the OCT features and our results correspond well with clinical observations

Despite limitations, the developed algorithms represent a useful application of a neural net using supervised learning of OCT images in neovascular AMD. Results demonstrate a useful capacity to recreate virtual visual acuity outcomes for a user-defined combination of physical characteristics. Future improvements could be made to automate measurements and consider incorporating other physical or clinical characteristics such as previous OCT appearances and vision as well as timing of intravitreal injections. The clinical and research applications of such algorithms include optimisation of treatment strategies, developing clinical trial endpoints and managing patient expectations in clinical practice. 


\section{Acknowledgements}

a. Funding/ Support: No external funding was provided in support of this work

b. Financial Disclosures: Tariq Aslam has received funding and educational grants from Bayer, Novartis, Laboratoires Thea, Oraya, Bausch and Lomb. No other author financial disclosures.

c. We acknowledge support from David Hoyle, Mathematician, Honorary Senior Lecturer, University of Manchester.

\section{Funding}

This research did not receive any specific grant from funding agencies in the public, commercial, or not-for-profit sectors.

\section{References}

1. Heier JS, Brown DM, Chong V, et al. Intravitreal Aflibercept (Vegf TrapEye) in Wet Age-Related Macular Degeneration. Ophthalmology 2012;119:2537-48. 2. Balaskas K, Karampelas M, Horani M, Hotu O, Keane P, Aslam T. Quantitative Analysis of Pigment Epithelial Detachment Response to Different AntiVascular Endothelial Growth Factor Agents in Wet Age-Related Macular Degeneration. Retina 2016; 37(7):1297-1304.

3. Arnold JJ, Markey CM, Kurstjens NP, Guymer RH. The Role of Sub-Retinal Fluid in Determining Treatment Outcomes in Patients with Neovascular Age-Related Macular Degeneration-a Phase IV Randomised Clinical Trial with Ranibizumab: The Fluid Study. BMC Ophthalmol 2016;16:31.

4. Wickremasinghe SS, Janakan V, Sandhu SS, Amirul-Islam FM, Abedi F, Guymer RH. Implication of Recurrent or Retained Fluid on Optical Coherence Tomography for Visual Acuity During Active Treatment of Neovascular AgeRelated Macular Degeneration with a Treat and Extend Protocol. Retina 2016;36:1331-9.

5. Caballero S, Swaney J, Moreno K, et al. Anti-Sphingosine-1-Phosphate Monoclonal Antibodies Inhibit Angiogenesis and Sub-Retinal Fibrosis in a Murine Model of Laser-Induced Choroidal Neovascularization. Exp Eye Res 2009;88:36777.

6. Keane PA, Patel PJ, Ouyang Y, et al. Effects of Retinal Morphology on Contrast Sensitivity and Reading Ability in Neovascular Age-Related Macular Degeneration. Invest Ophthalmol Vis Sci 2010;51:5431-7.

7. Jaffe GJ, Martin DF, Toth CA, et al. Macular Morphology and Visual Acuity in the Comparison of Age-Related Macular Degeneration Treatments Trials. Ophthalmology 2013;120:1860-70.

8. Moutray T, Alarbi M, Mahon G, Stevenson M, Chakravarthy U. Relationships between Clinical Measures of Visual Function, Fluorescein Angiographic and Optical Coherence Tomography Features in Patients with Subfoveal Choroidal Neovascularisation. Br J Ophthalmol 2008;92:361-4. 
9. Bloch SB, Lund-Andersen H, Sander B, Larsen M. Subfoveal Fibrosis in Eyes with Neovascular Age-Related Macular Degeneration Treated with Intravitreal Ranibizumab. Am J Ophthalmol 2013;156:116-124.

10. Zhang H, Yang Y, Takeda A, et al. A Novel Platelet-Activating Factor Receptor Antagonist Inhibits Choroidal Neovascularization and Subretinal Fibrosis. PLoS One 2013;8(6):e68173.

11. Ryu CL, Al-Humaid S, Rampakakis E, Galic IJ, Chen JC. Correlation of Visual Acuity with Fibrotic Scar Location in Treated Neovascular Age-Related Macular Degeneration Eyes. Retina 2016;36:1324-30.

12. Kuhn M. Manual for the Implementation of Neural Networks in Matlab. Norderstedt: Grin verlag, 2005:2-8.

13. Shereef IK, Baboo SS. An Efficient Weather Forecasting System Using Artificial Neural Network. IJESD 2010;1(4):321-326.

14. Marvin L. Neural Networks with Matlab. CreateSpace Independent Publishing Platform, 2016:1-5.

15. Peduzzi P, Concato J, Feinstein AR, Holford TR. Importance of Events Per Independent Variable in Proportional Hazards Regression Analysis. II. Accuracy and Precision of Regression Estimates. J Clin Epidemiol 1995;48:1503-10.

16. Agharezaei L, Agharezaei Z, Nemati A, et al. The Prediction of the Risk Level of Pulmonary Embolism and Deep Vein Thrombosis through Artificial Neural Network. Acta Inform Med 2016;24:354-359.

17. Razmara J, Zaboli MH. Elderly Fall Risk Prediction Based on a Physiological Profile Approach Using Artificial Neural Networks. Health Informatics J. doi: 10.1177/1460458216677841.2016.11.01

18. M.Shashi YR. Atmospheric Temperature Prediction Using Support Vector Machines. IJCTE 2009;1:1793-8201.

19. Patel PJ, Chen FK, Rubin GS, Tufail A. Intersession Repeatability of Visual Acuity Scores in Age-Related Macular Degeneration. Invest Ophthalmol Vis Sci 2008;49:4347-52.

20. Aslam T, Mahmood S, Balaskas K, et al. Repeatability of Visual Function Measures in Age-Related Macular Degeneration. Graefes Arch Clin Exp Ophthalmol 2014;252:201-6.

21. Schmidt-Erfurth U, Bogunovic H, Sadeghipour A, et al. Machine Learning to Analyze the Prognostic Value of Current Imaging Biomarkers in Neovascular AgeRelated Macular Degeneration. Ophthalmol Retina. doi.org/10.1016/ j.oret.2017.03.15

\section{Figure Legends}

Figure 1. Distribution of final errors between target and neural net test results. 
Figure 2. Three-dimensional plot of impact of OCT changes on visual acuity. The model plots impact of changes in in thickness of subretinal fluid and subretinal hyperreflective material when external limiting membrane is intact.

Figure 3. Three-dimensional plot of impact of OCT changes on visual acuity. The model plots impact of changes in in thickness of subretinal fluid and subretinal hyperreflective material when external limiting membrane is not intact.

Table 1. Table listing all 15 of the OCT-based features. The last column represents results from the forward feature selection algorithm which ranks the features according to the stage at which they were prioritised. Subretinal hyper-reflective material is determined the most important factor affecting vision. 
Table 1. Characteristics of OCT features used to develop the neural net and their rank importance.

\begin{tabular}{|c|c|c|c|c|c|}
\hline OCT Feature: & Minimum & Maximum & Mean & Median & $\begin{array}{l}\text { Step Added/ } \\
\text { Rank }\end{array}$ \\
\hline \multicolumn{6}{|l|}{$\begin{array}{l}\text { foveal contour (convex } \\
1 \text {,concave }-1 \text {, flat } 0 \text { ) }\end{array}$} \\
\hline & -1 & 1 & -0.74 & -1 & 13 \\
\hline Retinal thickness $(\mu \mathrm{m})$ & 21 & 808 & 171.27 & 158 & 7 \\
\hline $\begin{array}{l}\text { Subretinal hyperreflective } \\
\text { material }(\mu \mathrm{m})\end{array}$ & 0 & 1000 & 29.98 & 0 & 1 \\
\hline $\begin{array}{l}\text { Subretinal fluid } \\
(\mu \mathrm{m})\end{array}$ & 0 & 1184 & 23.68 & 0 & 5 \\
\hline $\begin{array}{l}\text { Sub RPE hyperreflective } \\
\text { material }(\mu \mathrm{m})\end{array}$ & 0 & 570 & 28.82 & 0 & 15 \\
\hline $\begin{array}{l}\text { Sub RPE fluid } \\
(\mu \mathrm{m})\end{array}$ & 0 & 748 & 29.54 & 0 & 3 \\
\hline $\begin{array}{l}\text { Choroid thickness } \\
(\mu \mathrm{m})\end{array}$ & 12 & 203 & 140.60 & 142 & 11 \\
\hline $\begin{array}{l}\text { Choroid shadow } \\
(\mu \mathrm{m})\end{array}$ & 0 & 256 & 135.74 & 135 & 8 \\
\hline $\begin{array}{l}\text { Central map thickness } \\
(\mu \mathrm{m})\end{array}$ & 62 & 1230 & 250.51 & 228 & 4 \\
\hline Map n $(\mu \mathrm{m})$ & 26 & 1163 & 290.84 & 275 & 9 \\
\hline Map e $(\mu \mathrm{m})$ & 133 & 1239 & 286.34 & 267 & 14 \\
\hline Map s $(\mu \mathrm{m})$ & 121 & 1068 & 286.97 & 273 & 10 \\
\hline Map w $(\mu \mathrm{m})$ & 162 & 1161 & 285.30 & 271 & 12 \\
\hline $\begin{array}{l}\text { ELM intact at foveolar } \\
\text { ( } 0=\text { no, } 1=\text { yes) }\end{array}$ & 0 & 1 & 0.42 & 0 & 2 \\
\hline $\begin{array}{l}\text { No of cysts in central foveal } \\
(2 \mathrm{~mm}) \text { zone }\end{array}$ & 0 & 23 & 0.86 & 0 & 6 \\
\hline
\end{tabular}


Test: $\mathbf{R}=\mathbf{0 . 8 5 2 3 9}$

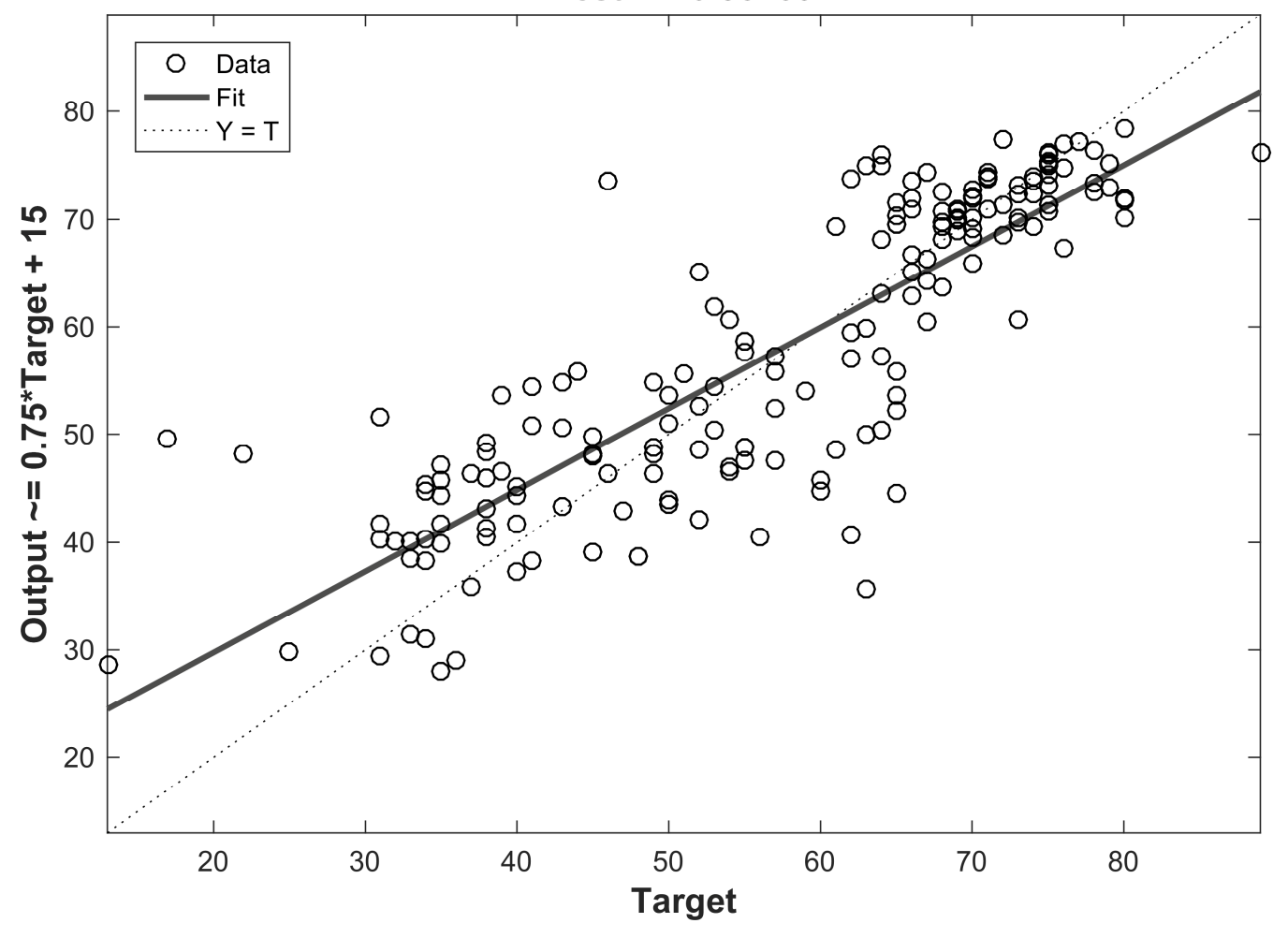




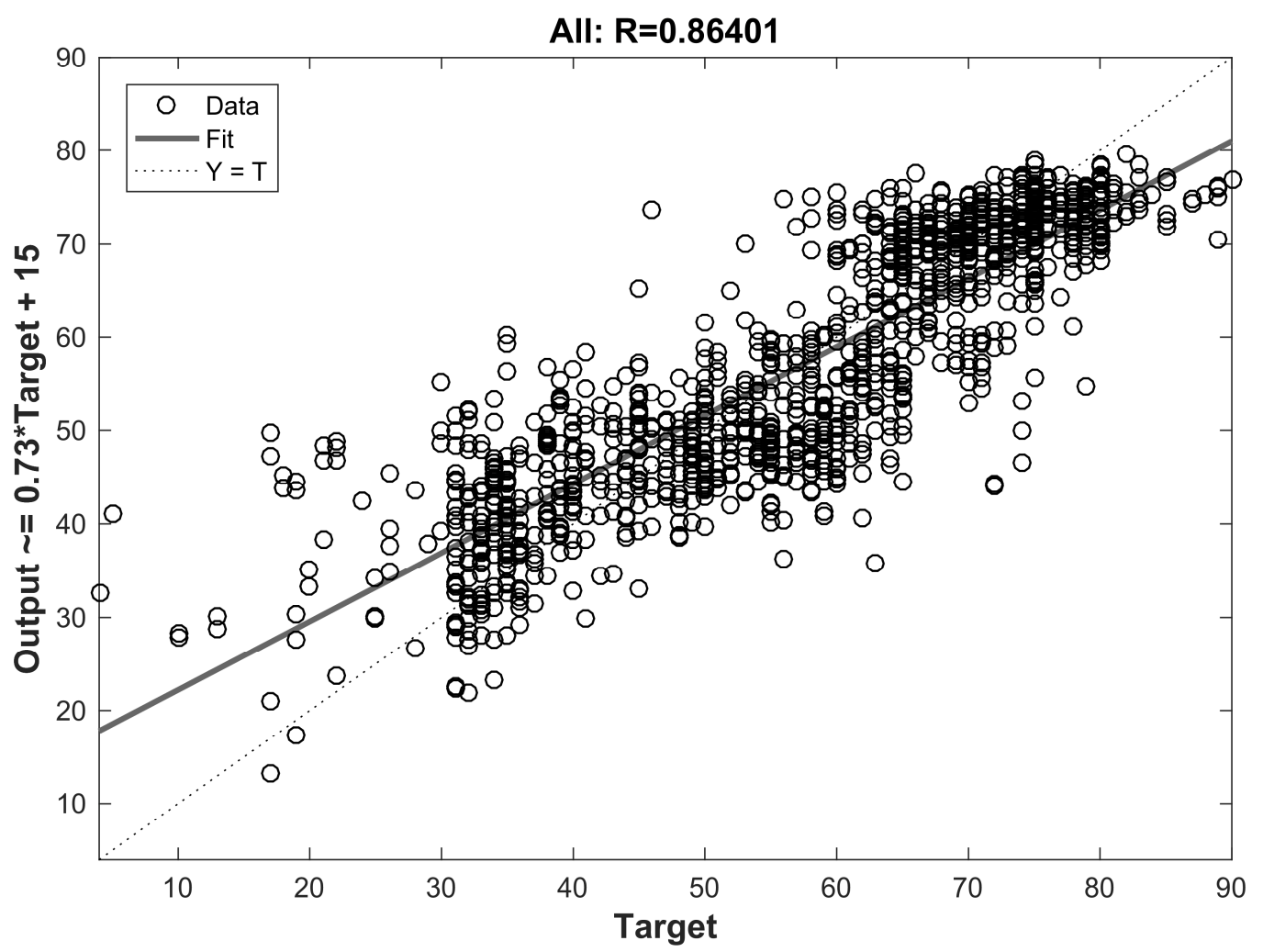




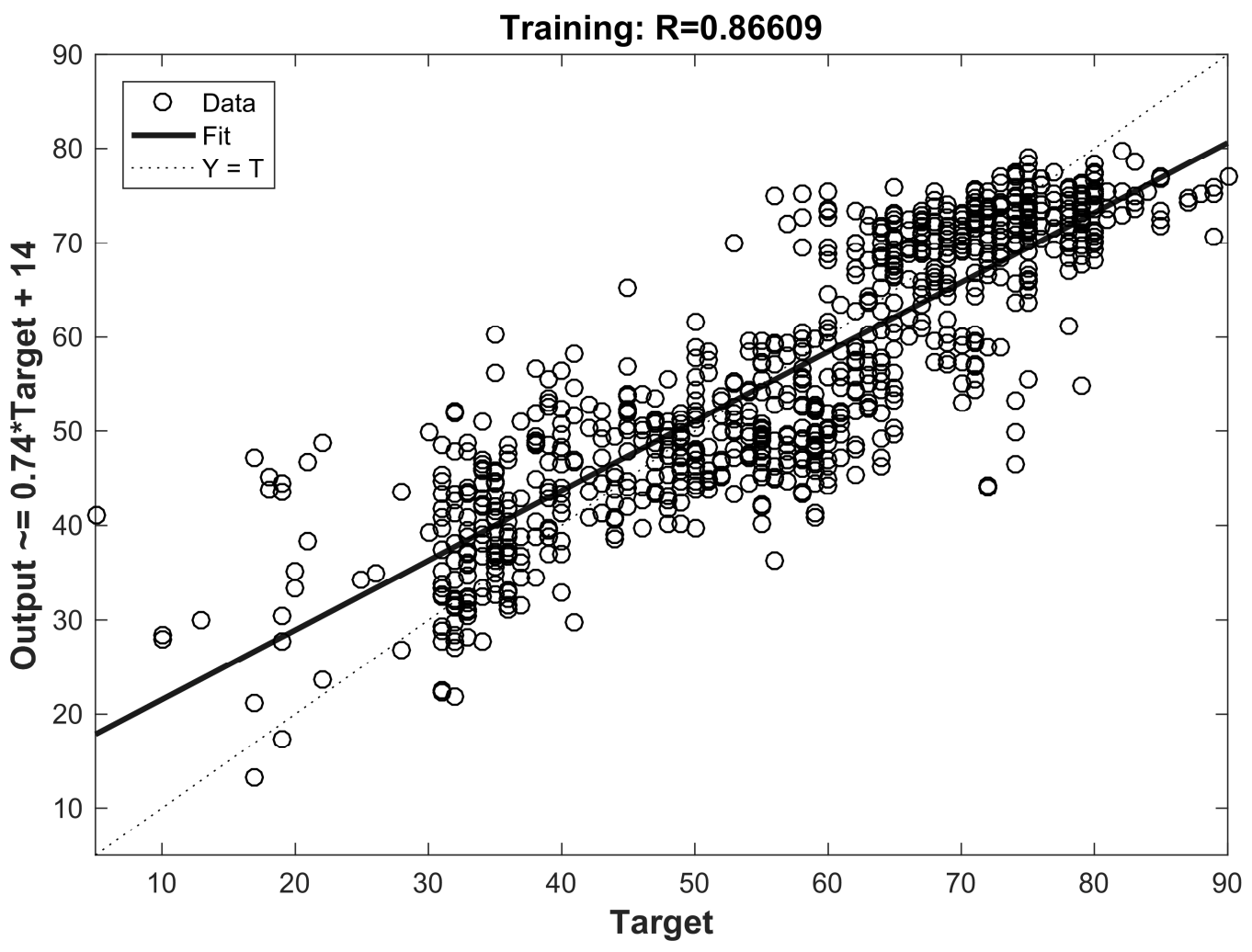




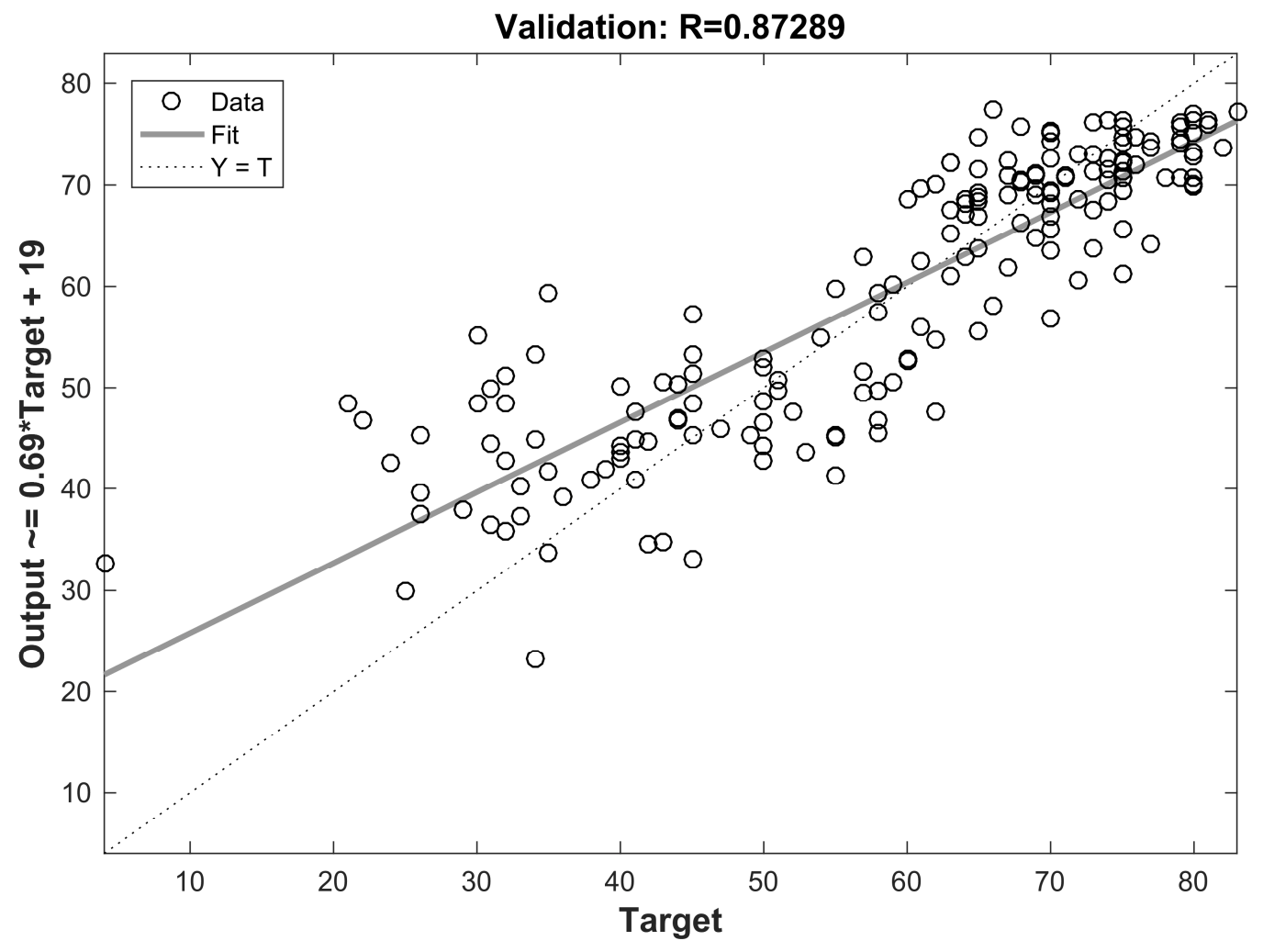


Artificial Intelligence-based Impact of OCT features on Vision

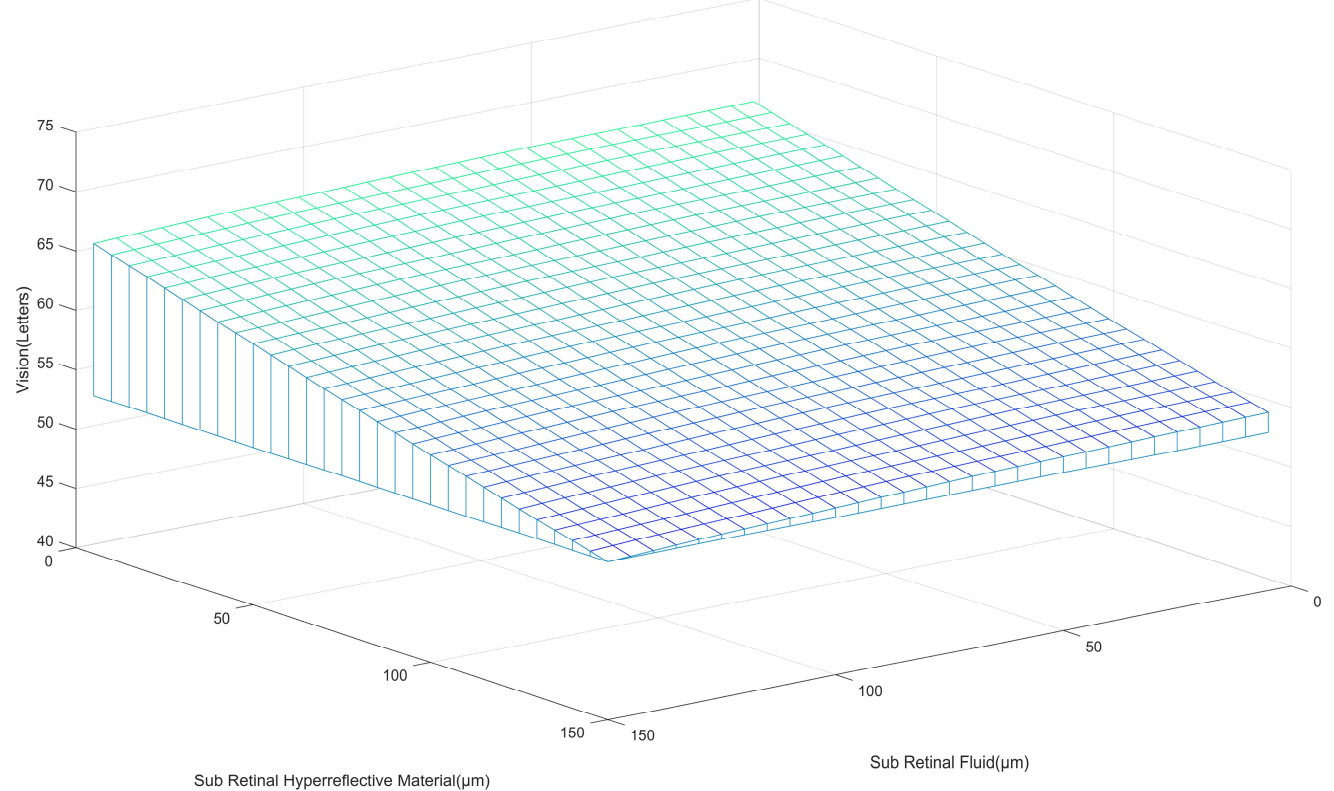


Artificial Intelligence-based Impact of OCT features on Vision

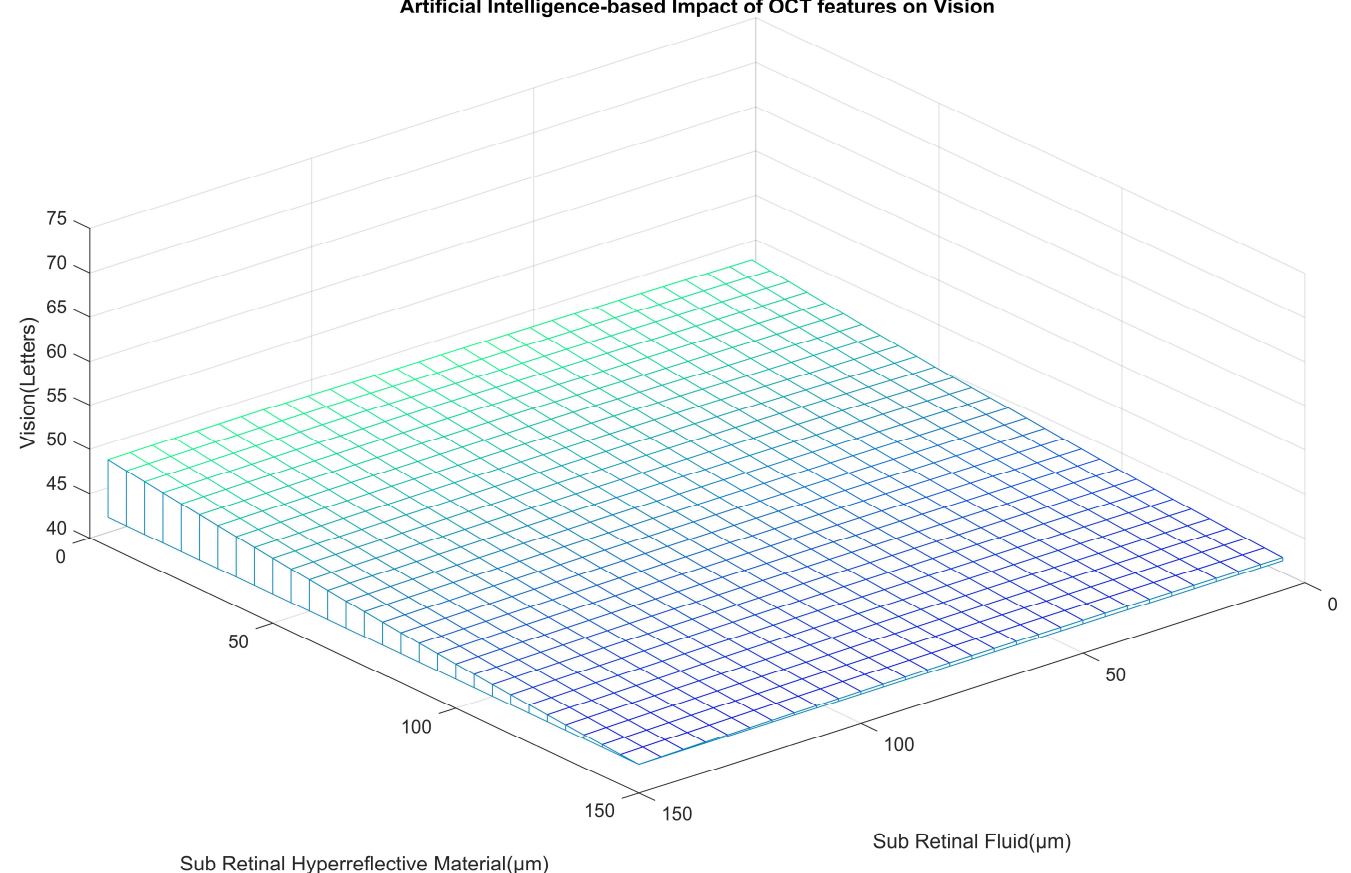

Sub Retinal Hyperreflective Material $(\mu \mathrm{m})$ 
AJO-17-1061, Use of a Neural Net to Model the Impact of Optical Coherence Tomography Abnormalities on Vision in Age-Related Macular Degeneration

\section{Summary Statement}

This paper addresses the relationship between structure and function in age-related macular degeneration by using a neural net to model the impact of optical coherence tomography changes on visual acuity. The results give useful clinical insight into the effect of different aspects of optical coherence tomography change on vision. 